\title{
LA FAUNE DU PLÉISTOCÈNE SUPÉRIEUR - HOLOCÈNE ANCIEN DE LA TOCA DA PENA (PIAUÍ, BRÉSIL) - ÉTUDE PALÉONTOLOGIQUE.
}

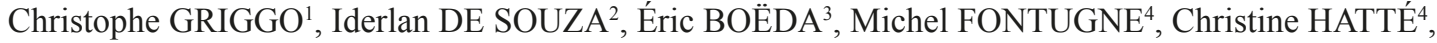 \\ Antoine LOURDEAU ${ }^{5} \&$ Niède GUIDON ${ }^{6}$
}

\section{RÉSUMÉ}

Le site de la Toca da Pena correspond à une petite grotte qui se trouve dans le massif calcaire d'Antero, au sud de la Serra da Capivara et à $7 \mathrm{~km}$ à l'est-nord-est de la ville de Coronel José Dias. Des fouilles entreprises entre 2008 et 2014 ont permis de recueillir, associés à quelques artefacts lithiques, de nombreux vestiges osseux. Près de la moitié d'entre eux sont rapportés à Scelidodon piauiense, un paresseux terrestre de la taille d'un grand bœuf. Ce Mylodontidé est associé à Leopardus pardalis, Hippidion, Tayassu pecari, Mazama gouazoubira, Mazama americana et à Palaeolama niedae.

Cette association faunique permet de dater ce site du Pléistocène supérieur ou du tout début de l'Holocène. Par ailleurs, cette faune de la Toca da Pena présente beaucoup de similitude avec celle de la Toca do Barrigudo, qui se trouve à moins d'un kilomètre et d'où provient l'holotype de l'espèce : Scelidodon piauiense Guérin \& Faure, 2004.

Mots-clés : Xenarthra, Scelidodon, Mylodontidae, Scelidotheriinae, Toca da Pena, Piauí, Brésil, Pléistocène supérieur, Holocène ancien, Palaeolama, Mazama

\section{ABSTRACT}

THE UPPER PLEISTOCENE - EARLY HOLOCENE FAUNA OF TOCA DA PENA (PIAUÍ, BRAZIL) - PALEONTOLOGICAL STUDY

The site of Toca da Pena corresponds to a small cave located in the limestone massif of Antero, south of the Serra de Capivara and $7 \mathrm{~km}$ to the east-north-east of the town of Coronel José Dias. Excavations, undertaken between 2008 and 2014 , together with some lithic artefacts, have yielded numerous osseous remains. Almost half of them are reported to Scelidodon piauiense, a ground sloth which was the size of a large cattle. This Mylodontidae is associated with Leopardus pardalis, Hippidion, Tayassu pecari, Mazama gouazoubira, Mazama americana and Palaeolama niedae.

This faunal association allows us to date this site of the Upper Pleistocene or the Early Holocene. Moreover, the Toca da Pena fauna is very similar to that of Toca do Barrigudo, which is less than one kilometer away and yielded the holotype of the species: Scelidodon piauiense Guérin \& Faure, 2004.

Keywords: Xenarthra, Scelidodon, Mylodontidae, Scelidotheriinae, Toca da Pena, Piauí, Brazil, Upper Pleistocene, Early Holocene, Palaeolama, Mazama

\section{1 - INTRODUCTION}

La Toca da Pena (ou grotte de la Plume) est située dans l'État du Piauí, au sud du Parc National Serra da Capivara et plus précisément au pied du massif d'Antero, à environ $7 \mathrm{~km}$ à l'est-nord-est de la ville de Coronel José Dias (fig. 1). Ce site, découvert en 2008, a été fouillé jusqu'en 2014 par une mission francobrésilienne, dans le cadre du projet sur « Le peuplement pléistocène et holocène du Nordeste - Brésil » dirigé par
E. Boëda. C'est un site mixte, à la fois grotte et abri sous roche (fig. 2), qui a livré quelques artefacts lithiques en association stratigraphique avec de nombreux restes fauniques.

C. Guérin, lors d'une étude préliminaire réalisée en 2009, fournit une première liste des espèces présentes dans la Toca da Pena : Scelidodon piauiense, Mazama, Blastocerus dichotomus, Palaeolama, un Tayassuidé, un Félidé, un petit Canidé, des Dasypodidés et des restes de tortue (Rasse et al., 2009 ; Guérin \& Faure, 2014).

\footnotetext{
${ }^{1}$ Université Grenoble Alpes, Laboratoire EDYTEM - UMR 5204, Campus scientifique, Bâtiment Pôle montagne, FR-73376 LE BOURGETDU-LAC cedex. Courriel : Christophe.Griggo@univ-grenoble-alpes.fr

${ }^{2}$ Universidade Federal do Vale do São Francisco, Campus Serra da Capivara, Arqueologia e preservação patrimonial, BR-64770-000 SÃO RAIMUNDO NONATO, Piaú, Brasil. Courriel : iderlanz@hotmail.com

${ }^{3}$ Université Paris Ouest Nanterre, Laboratoire d'Anthropologie des Techniques, des Espaces et des Territoires au Pliocène et Pléistocène - UMR 7041 CNRS ArScAn, Maison René Ginouvès, 21, allée de 1'Université, FR-92023 NANTERRE cedex. Courriel : boeda.eric@gmail.com

${ }^{4}$ Université Paris Saclay, Laboratoire des Sciences du Climat et de l'Environnement - UMR 8212, CEA-Orme des Merisiers, FR-91191 GIFSUR-YVETTE. Courriels : michel.fontugne@1sce.ipsl.fr, christine.hatte@1sce.ipsl.fr

${ }_{5}^{5}$ Muséum national d'Histoire naturelle - Département de Préhistoire, UMR 7194, IPH - 1, rue René Panhard, FR-75013 PARIS

Courriel : antoine.lourdeau@mnhn.f

${ }^{6}$ Fundação Museu do Homem Americano (FUMDHAM), BR-64770-000, SÃO RAIMUNDO NONATO, Piauí, Brasil. Courriel : guidon@fumdham.org.br
} 


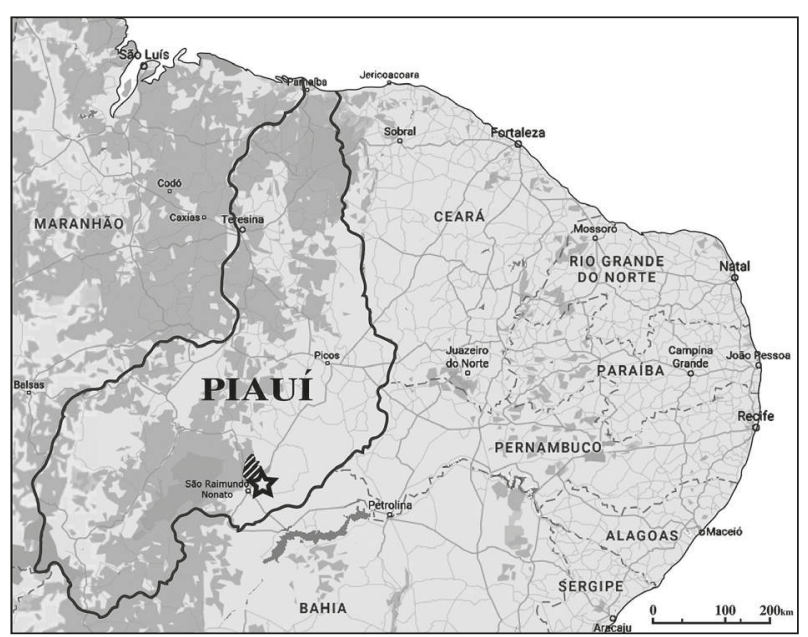

Fig. 1 : Carte de localisation du site de la Toca de Pena, dans le Parc National de la Serra da Capivara (Piauí).

Fig. 1: Location of the site of Toca da Pena, in Serra da Capivara National Park (Piaui).

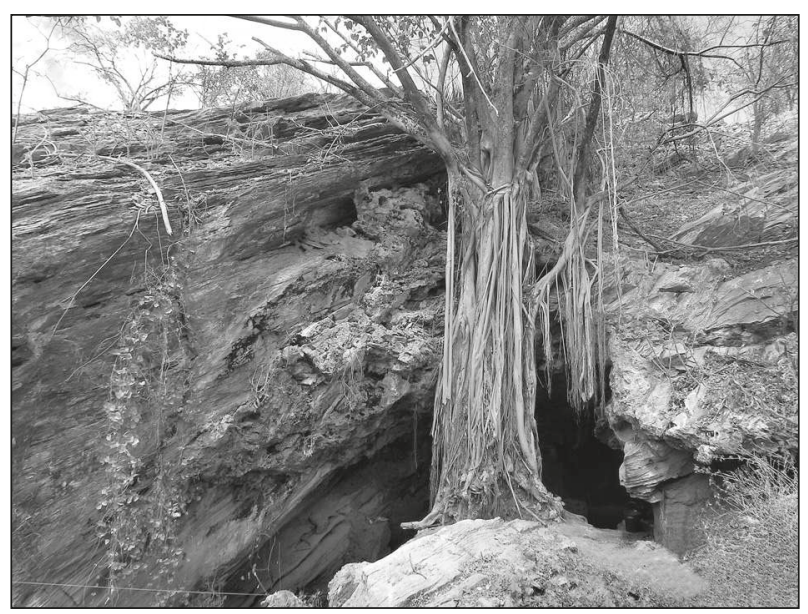

Nous avons examiné l'ensemble des vestiges osseux recueillis dans la Toca da Pena entre 2008 et 2014. Cela correspond à un total de 1946 restes se rapportant à un amphibien, trois reptiles et quinze mammifères. Parmi ces derniers, c'est un paresseux terrestre appartenant à la famille des Mylodontidés, Scelidodon piauiense, qui a livré le plus grand nombre de restes. Il est associé à l'ocelot, Leopardus pardalis, à un Equidé, Hippidion, au pécari à lèvres blanches, Tayassu pecari, au daguet gris, Mazama gouazoubira, au grand daguet, Mazama americana et à un Camélidé, Palaeolama niedae, parmi les espèces les plus caractéristiques (tab. 1).

Nous proposons dans le cadre de cet article de faire l'étude paléontologique de cette faune de la Toca da Pena, qui présente de grande similitude avec le site, tout proche, de la Toca do Barrigudo où a été défini l'espèce Scelidodon piauiense, par C. Guérin et M. Faure en 2004.

\section{2 - LE MYLODONTIDÉ - SCELIDODON PIAUIENSE}

Dans le site de la Toca da Pena, Scelidodon piauiense est l'espèce la mieux représentée. Ce paresseux terrestre, de la taille d'un grand bœuf, est un Xénarthré, Mylodontidé de la sous-famille des Scélidothériinés. Le genre Scelidodon, reconnu dans toute l'Amérique du Sud, dans différents gisements datant du Pléistocène moyen au Pléistocène supérieur, compte actuellement trois espèces (Guérin \& Faure, 2004) :

- Scelidodon tarijensis (H. Gervais \& F. Ameghino, 1880), défini dans le Pléistocène moyen de Bolivie, du Chili, d'Uruguay et d'Argentine, est de grande taille ;

Fig. 2 : Entrée de la Toca da Pena.

Fig. 2: Entrance to the Toca da Pena.

\begin{tabular}{|c|c|c|c|c|c|}
\hline \multirow{2}{*}{\multicolumn{3}{|c|}{ Fig. 2: Entrance to the Toca da Pena. }} & \multirow{3}{*}{$\begin{array}{c}\begin{array}{c}\text { Secteurs } \\
\text { devant grotte }\end{array} \\
17 \\
\end{array}$} & \multirow{3}{*}{$\begin{array}{c}\text { Secteur grotte } \\
29 \\
\end{array}$} & \multirow{3}{*}{$\begin{array}{c}\text { Total } \\
46\end{array}$} \\
\hline & & & & & \\
\hline Amphibia & Anura & Crapaud indéterminé & & & \\
\hline \multirow[t]{4}{*}{ Reptilia } & Testutines & Chelonoidis sp. & 81 & 18 & 99 \\
\hline & Squamata & Iguana iguana & 0 & 2 & 2 \\
\hline & & non identifiés & 13 & 1 & 14 \\
\hline & Crocodilia & Caiman sp. & 1 & 3 & 4 \\
\hline \multirow[t]{16}{*}{ Mammalia } & Dasypodidae & Euphractus sexcinctus & 15 & 21 & 36 \\
\hline & & Tolypeutes tricinctus & 10 & 7 & 17 \\
\hline & & Dasypus novemcinctus & 1 & 2 & 3 \\
\hline & Mylodontidae & Scelidodon piauiense & 120 & 730 & 850 \\
\hline & Caviidae & Kerodon rupestris & 43 & 47 & 90 \\
\hline & Erethizontidae & Coendou prehensilis & 1 & 3 & 4 \\
\hline & Echimyidae & Thrichomys apereoides & 1 & & 1 \\
\hline & Agoutiidae & Agouti paca & 1 & & 1 \\
\hline & Felidae & Leopardus pardalis & 59 & 13 & 72 \\
\hline & & Félin de petite taille & 10 & 6 & 16 \\
\hline & Equidae & Hippidion sp. & 4 & & 4 \\
\hline & Tayassuidae & Tayassu pecari & 16 & 10 & 26 \\
\hline & Cervidae & Mazama americana & 11 & 5 & 16 \\
\hline & & Mazana gouazoubira & 3 & 11 & 14 \\
\hline & Camelidae & Palaeolama niedae & 4 & 21 & 25 \\
\hline & Ungulata indéterminés & & 16 & 14 & 30 \\
\hline \multicolumn{2}{|c|}{ Microfaune indéterminée } & & 454 & 122 & 576 \\
\hline \multicolumn{2}{|c|}{ Total par taxon } & & 881 & 1065 & 1946 \\
\hline
\end{tabular}

Tab. 1 : Toca da Pena - Répartition des restes déterminés par taxon et par secteur.

Tab. 1: Toca da Pena-Distribution of determined remains per taxon and per sector. 


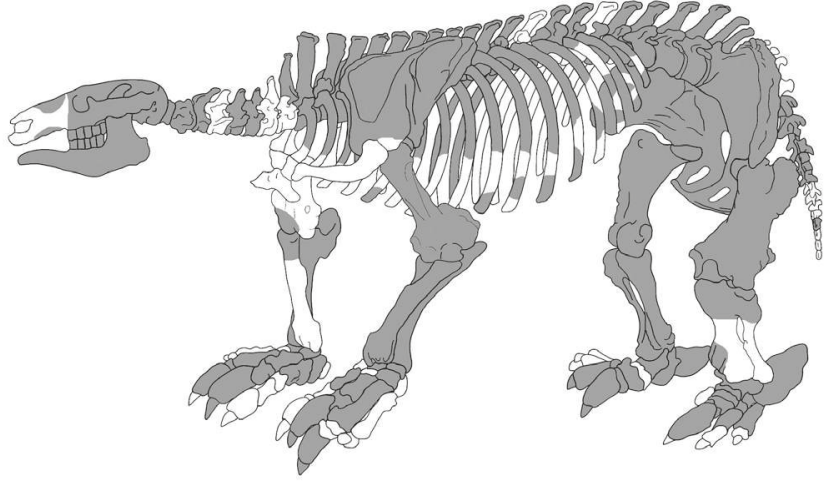

Vue antéro-latérale gauche

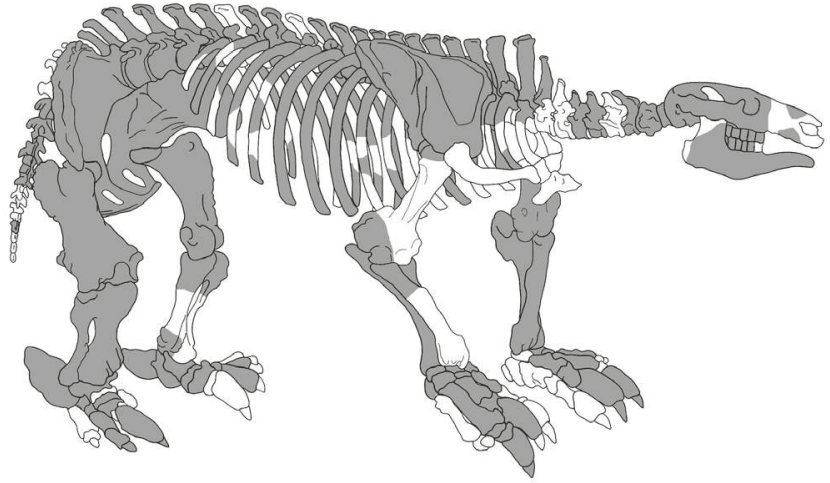

Vue antéro-latérale droite

Fig. 3 : Représentation des éléments squelettiques présents pour Scelidodon - spécimen nº 1.

Fig. 3: Representation of skeletal elements found for Scelidodon-specimen $n^{\circ} 1$.

- Scelidodon chiliensis (Lydekker, 1886), reconnu dans des gisements pléistocènes supérieurs du Chili, du Pérou, de Bolivie et d'Equateur, se caractérise par une taille un tiers plus petit que le précédent et par des molariformes dépourvues de sillon médian;

- Scelidodon piauiense Guérin \& Faure, 2004, identifié seulement dans le Nordeste du Brésil, dans l'état du Piauí, est de taille équivalente à Scelidodon chiliensis, mais présente des proportions et une morphologie crânienne différentes. Sa présence est contemporaine du Pléistocène supérieur et de l'Holocène ancien.

Par ailleurs le genre Scelidodon est proche du genre Catonyx qui appartient à la même sous-famille et est également présent dans les sites du Nordeste du Brésil, datant du Pléistocène supérieur (Guérin \& Faure, 2004).

Dans le site de la Toca da Pena, nous disposons actuellement de 850 restes osseux attribués au Scelidodon. Ils proviennent pour l'essentiel des squelettes de deux individus :

- le spécimen $n^{\circ} 1$, dont la majorité des ossements ont été recueillis dans la grotte, dans la partie superficielle du remplissage. Beaucoup étaient encore en connexion anatomique partielle. Il s'agit d'un animal adulte dont tous les os sont épiphysés. La plupart des os ont été recueillis (fig. 3) et ils présentent un bon état de conservation ;
- le spécimen $\mathrm{n}^{\circ} 2$, qui correspond à un animal juvénile : les épiphyses des os longs ne sont pas soudées aux corps diaphysaires. Il n'est représenté que par quelques ossements. Ils ont été trouvés dispersés dans la moitié inférieure de la séquence stratigraphique fouillée. Ils sont comparables, d'un point de vue morphologique, à ceux du spécimen $n^{\circ} 1$ (cf. infra). Cependant, bien que cet animal n'avait pas fini sa croissance au moment de sa mort, il est plus grand que le spécimen $n^{\circ} 1$.

Cette étude paléontologique est focalisée sur une analyse morphologique et biométrique des os complets se rapportant au spécimen $n^{\circ} 1$, qui correspond à un adulte. Nous insisterons plus particulièrement sur l'étude du crâne, de la mandibule, des dents et de l'humérus afin de les comparer au type de l'espèce Scelidodon piauiense. Cette espèce a été définie par Guérin et Faure en 2004, à partir d'un squelette subcomplet recueilli dans la Toca do Barrigudo, qui se trouve à moins de $500 \mathrm{~m}$ de la Toca da Pena. En nous référant aux travaux de Pujos (2000) et de Guérin \& Faure (2004), ces éléments squelettiques ont également été comparés avec les os homologues de S. chiliensis.

Lors d'un séjour à la Fundação Museu do Homem americano (FUMDHAM), à São Raimundo Nonato, nous avons pu comparer directement le Scelidodon de la Toca da Pena avec l'holotype de l'espèce Scelidodon
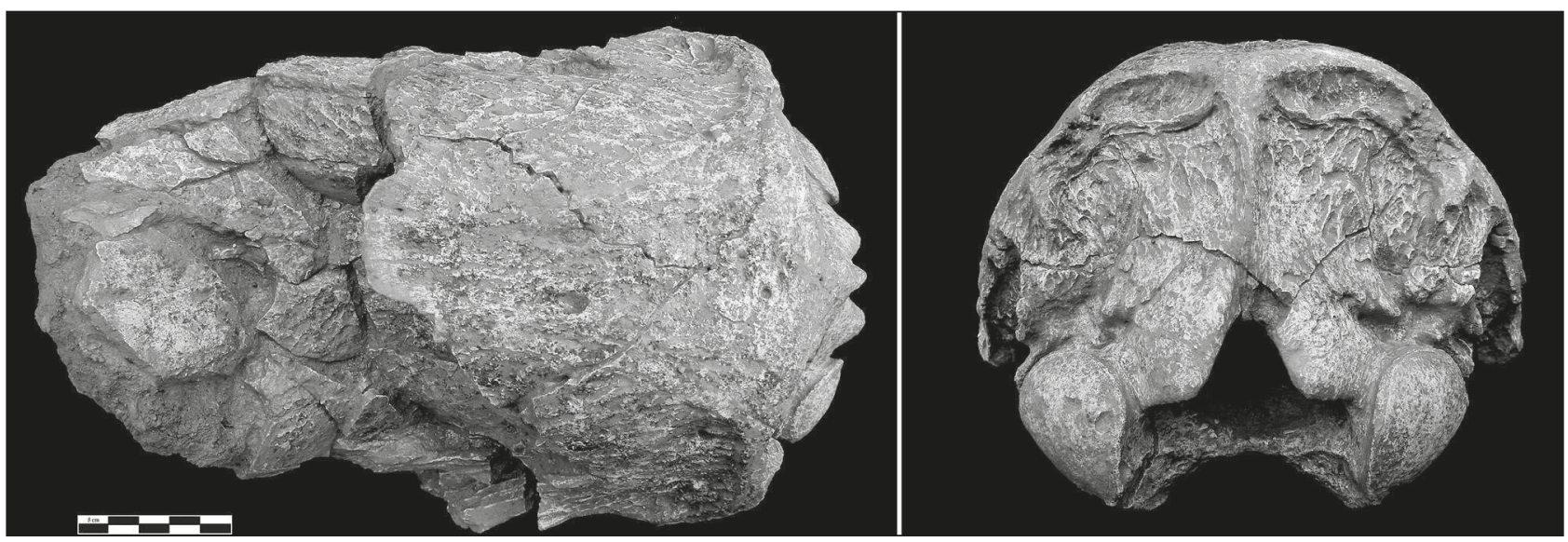

Fig. 4 : Crâne de Scelidodon - spécimen n¹ (FUMDHAM-n²35041). à gauche : vue dorsale ; à droite : vue caudale Fig. 4: Skull of Scelidodon - specimen $n^{\circ} 1$ (FUMDHAM- $\left.{ }^{\circ} 235041\right)$ - left: dorsal view; right: caudal view. 


\begin{tabular}{|c|c|c|c|c|c|}
\hline \multirow[b]{2}{*}{ Crâne } & \multicolumn{2}{|c|}{ Scelidodon piauiense } & \multicolumn{3}{|c|}{$\begin{array}{l}\text { Scelidodon chiliensis } \\
\quad(\text { Pujos, 2000) }\end{array}$} \\
\hline & $\begin{array}{c}\text { Toca da pena } \\
\text { Brésil }\end{array}$ & $\begin{array}{c}\text { Toca do } \\
\text { Barrigudo } \\
\text { Brésil }\end{array}$ & $\begin{array}{c}\text { Pampa de } \\
\text { los Fosiles } \\
\text { Pérou }\end{array}$ & $\begin{array}{c}\text { Chingas } \\
\text { Pérou }\end{array}$ & $\begin{array}{c}\text { Tamarugal } \\
\text { Chili }\end{array}$ \\
\hline Hauteur face occipitale à partir bord sup. du foramen magnum & 56,3 & 64,7 & 79 & 77 & 72 à 74,5 \\
\hline Hauteur face occipitale à partir bord inf. du foramen magnum & 85,2 & 99,7 & 105 & 95 & 88 à 88,5 \\
\hline Hauteur totale face occipitale (condyle-crête occipitale) & 95,5 & 110,6 & 115 & - & - \\
\hline Largeur face occipitale au niveau des apophyses mastoïdes & 142,4 & 145,3 & 152,5 & 152 & 149 à 152,5 \\
\hline Diamètre transversal pris à l'extérieur des condyles occipitaux & 95,4 & 94,3 & 118 & 104 & 99 à 103 \\
\hline
\end{tabular}

Tab. 2 : Scelidodon - Comparaison des mesures du crâne - dimensions en mm. Tab. 2: Scelidodon-Comparison of the measurements of skull-dimensions in mm.

piauiense, ainsi qu'avec quelques os longs de Scelidodon piauiense et de Catonyx cuvieri, provenant de la Toca do Garrincho (située à $10 \mathrm{~km}$ à l'ouest de la Toca da Pena). Par ailleurs, cela nous a permis d'observer, sur le fémur, quelques critères morphologiques permettant de distinguer Scelidodon de Catonyx.

\section{1 - LE CRÂNE}

Le crâne du spécimen $n^{\circ} 1$ de la Toca da Pena est incomplet. Il manque toute la région faciale. La zone frontale, au niveau des orbites, présente un important enfoncement. Mais les zones pariétales, temporales et la région occipitale sont parfaitement bien conservées, sans aucune déformation (fig. 4). Par conséquent, seule la face occipitale a pu faire l'objet d'une étude morphologique et biométrique (tab. 2).

En vue occipitale, le crâne de la Toca da Pena est plus bas que celui se rapportant à l'espèce $S$. chiliensis et se rapproche davantage, pour ce point, de celui de S. piauiense. Il est cependant, dans ses proportions générales, plus petit.

\section{2 - LES DENTS ET LES ÉLÉMENTS DE LA} MÂCHOIRE

Pour le spécimen $n^{\circ} 1$ de la Toca da Pena, nous disposons de quelques molariformes isolées, d'un fragment de maxillaire ne possédant qu'une seule molariforme, ainsi que des branches horizontales des deux hémi-mandibules. Pour celle de gauche, la série dentaire est complète (fig. 5). En revanche, il manque la première molariforme pour l'hémi-mandibule droite.

Les molariformes supérieures présentent un sillon médian bien marqué, ce qui leur confère une section trilobée. Cette morphologie dentaire est caractéristique de l'espèce Scelidodon piauiense (Guérin \& Faure, 2004).

Les longueurs des séries dentaires inférieures des mandibules du Scelidodon de la Toca da Pena sont comparables à celles de $S$. piauiense de la Toca do Barrigudo et par leurs proportions, ces mandibules se rapprochent davantage de celles de cette espèce que de S. chiliensis (tab. 3). De ce fait, la valeur du rapport : hauteur de la branche horizontale/longueur de la série

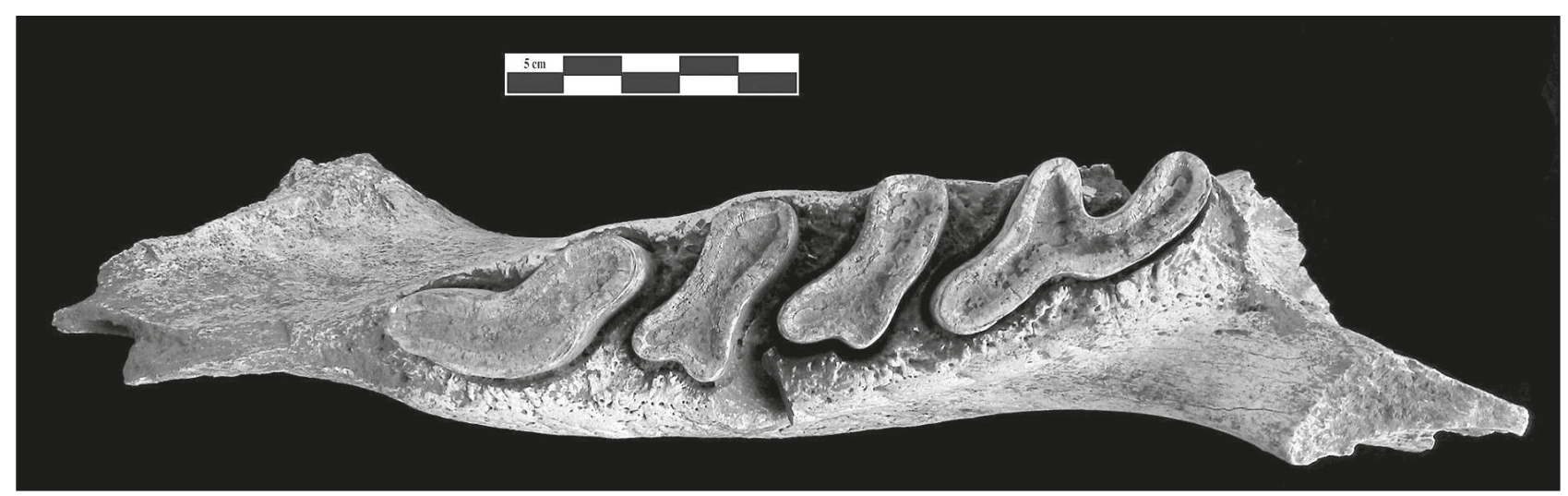

Fig. 5 : Mandibule gauche de Scelidodon - spécimen $n^{\circ} 1$ (FUMDHAM-n ${ }^{\circ 180468)}$ - vue occlusale.

Fig. 5: Left mandible of Scelidodon - specimen $n^{\circ} 1$ (FUMDHAM- $\left.n^{\circ} 180468\right)$ - occlusal view.

\begin{tabular}{|l|c|c|c|c|c|}
\cline { 2 - 6 } \multicolumn{1}{c|}{} & \multicolumn{3}{c|}{ Scelidodon piauiense } & \multicolumn{2}{c|}{ Scelidodon chiliensis } \\
\hline \multirow{2}{*}{ Mandibule } & \multicolumn{2}{|c|}{$\begin{array}{c}\text { (Guérin \& Faure, } \\
\text { 2004) }\end{array}$} & \multicolumn{2}{c|}{ (Pujos, 2000) } \\
\cline { 2 - 6 } & $\begin{array}{c}\text { Toca da Pena } \\
\text { (180468) } \\
\text { Brésil }\end{array}$ & $\begin{array}{c}\text { Toca da Pena } \\
(18049) \\
\text { Brésil }\end{array}$ & $\begin{array}{c}\text { Toca do } \\
\text { Barrigudo } \\
\text { Brésil }\end{array}$ & $\begin{array}{c}\text { Chingas } \\
\text { Pérou }\end{array}$ & $\begin{array}{c}\text { Pampa de } \\
\text { los Fossiles } \\
\text { Pérou }\end{array}$ \\
\hline Hauteur branche hori. Entre $\mathrm{M}_{3}-\mathrm{M}_{4}$ & 76,9 & - & 84,5 & 71 & 82 \\
Diamètre transversal branche hori. Entre $\mathrm{M}_{3}-\mathrm{M}_{4}$ & 33,2 & 35,4 & 34 & 31,5 & 33 \\
Longueur série dentaire inférieure & 98,7 & $98(?)$ & 98,1 & 105 & 127 \\
Rapport Haut. branche hori/long. série dentaire & 77,9 & --- & 86,1 & 67,6 & 64,6 \\
\hline
\end{tabular}

Tab. 3 : Scelidodon - Comparaison des mesures de la mandibule - dimensions en mm.

Tab. 3: Scelidodon-Comparison of the measurements of mandible-dimensions in mm. 
dentaire, obtenue pour le spécimen de la Toca da Pena se situe en position intermédiaire entre les deux espèces.

\section{3 - L'HUMÉRUS}

L’humérus du spécimen $n^{\circ} 1$ de la Toca da Pena présente un condyle proximal sphérique, bien dégagé et situé en position axiale. La face antérieure de la diaphyse est parcourue par de fortes crêtes d'insertions musculaires. L'épiphyse distale est très courte. Cette morphologie est caractéristique de la sous-famille des Scelidotheriinae.

Par ailleurs, l'humérus de la Toca da Pena présente un foramen antépicondylien au niveau de l'épiphyse distale (fig. 6). Ce caractère morphologique est typique du genre Scelidodon et permet de rejeter l'hypothèse d'une attribution au genre Catonyx. En effet, chez ce dernier, il n'y a pas de foramen antépicondylien.

Guérin \& Faure (2004) ont mis en évidence que Scelidodon piauiense se distingue de $S$. chiliensis par un humérus présentant des dimensions et des proportions sensiblement plus grandes (tab. 4).

Cependant un des humérus de $S$. chiliensis, mesuré par ces auteurs, est bien plus petit que les deux autres (tab. 4).
Selon Guérin (communication orale, 2014), cela pourrait être le reflet d'un très fort dimorphisme sexuel chez le genre Scelidodon.

L'humérus du spécimen $n^{\circ} 1$ de la Toca da Pena est bien plus petit que ceux de $S$. pianiense, mais il est plus grand que le plus petit humérus rapporté à $S$. chiliensis. Nous considérons donc que le Scelidodon de la Toca da Pena correspond à l'espèce Scelidodon piauiense, mais que, de par sa petite taille, il s'agit très certainement d'un individu femelle.

\section{4 - LE FÉMUR}

Les deux fémurs du spécimen $n^{\circ} 1$ ont été retrouvés lors des fouilles de la Toca da Pena. Ils ont été comparés avec des fémurs de Scelidodon piauiense et de Catonyx cuvieri (provenant de sites voisins et actuellement conservés dans les collections de la FUMDHAM à São Raimundo Nonato) et avec un fémur de $S$. chiliensis de la Pampa de los Fósiles (Pérou) (Pujos, 2000) (tab. 5).

Contrairement à ce qui a été noté par Guérin et Faure pour les humérus de $S$. piauiense provenant de la Toca do Barrigudo et de la Toca do Garrincho, qui sont
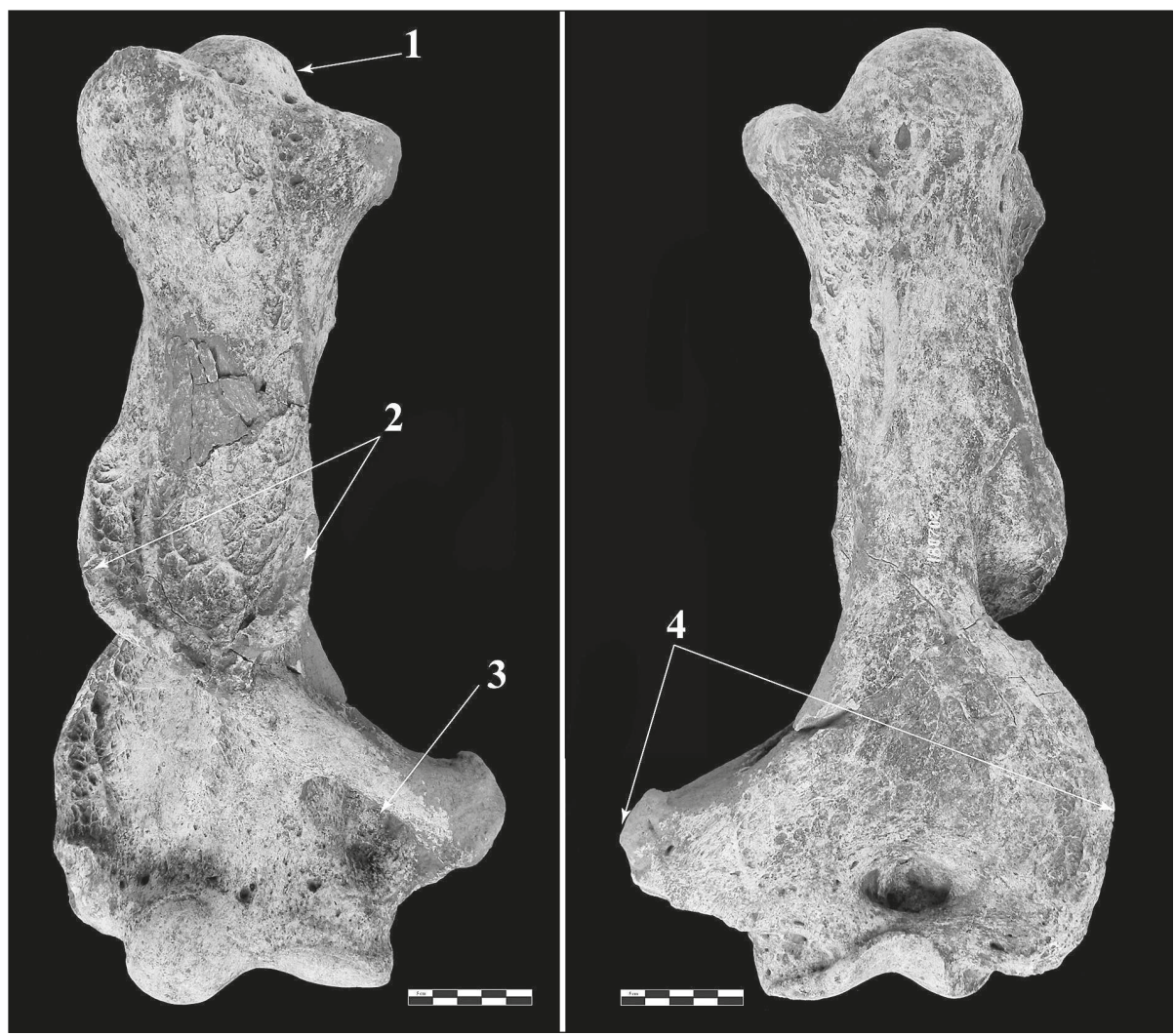

Fig. 6 : Humérus gauche de Scelidodonspécimen $n^{\circ} 1$ (FUMDHAM-n $\left.{ }^{\circ} 180702\right)$. À gauche : vue antérieure ; à droite : vue postérieure. 1/ condyle proximal sphérique ; 2/ fortes crêtes le long de la diaphyse ; 3/ présence d'un foramen épicondylien ; 4/ extrémité distale très large.

Fig. 6: Left humerus of Scelidodon specimen $n^{\circ} 1$ (FUMDHAM- $\left.{ }^{\circ} 180702\right)$ Left: anterior view; Right: posterior view. 1/ spherical proximal condyle; 2/ strong ridges along the diaphysis; 3/ presence of an epicondylian foramen; 4/ very wide distal end.

\begin{tabular}{|c|c|c|c|c|}
\hline \multicolumn{3}{|l|}{ Humérus } & Longueur totale & $\begin{array}{l}\text { Rapport longueur/ } \\
\text { diamètre transversal } \\
\text { maximal distal }\end{array}$ \\
\hline \multirow{3}{*}{ Scelidodon piauiense } & & $\begin{array}{c}\text { Toca da Pena - Brésil } \\
(180702)\end{array}$ & 345,3 & 2 \\
\hline & \multirow{2}{*}{ (Guérin \& Faure, 2004) } & $\begin{array}{c}\text { Toca do Barrigudo - Brésil } \\
(79142)\end{array}$ & 406 & 2,08 \\
\hline & & Toca do Garrincho - Brésil & 407 & 2,19 \\
\hline \multirow{3}{*}{ Scelidodon chiliensis } & \multirow{3}{*}{ (Pujos, 2000) } & \multirow{3}{*}{ Tamarugal - Chili } & 338 & 1,98 \\
\hline & & & 370 & 2 \\
\hline & & & 391 & - \\
\hline
\end{tabular}




\begin{tabular}{|c|c|c|c|c|c|c|}
\hline \multirow[b]{2}{*}{ Fémur } & \multicolumn{4}{|c|}{ Scelidodon piauiense } & \multirow{2}{*}{\begin{tabular}{|c|}
$\begin{array}{c}\text { Scelidodon chiliensis } \\
\text { (Pujos, 2000) }\end{array}$ \\
$\begin{array}{l}\text { Pampa de } \\
\text { los Fosiles } \\
\text { Pérou }\end{array}$ \\
\end{tabular}} & \multirow{2}{*}{$\begin{array}{l}\text { Catonyx cuvieri } \\
\begin{array}{c}\text { Toca do Garrincho } \\
(37496) \\
\text { Brésil }\end{array}\end{array}$} \\
\hline & $\begin{array}{c}\text { Toca da Pena } \\
\text { (234061) } \\
\text { Brésil }\end{array}$ & $\begin{array}{c}\text { Toca da Pena } \\
\text { (194759) } \\
\text { Brésil }\end{array}$ & $\begin{array}{c}\text { Toca do Barrigudo } \\
\text { (37565) } \\
\text { Brésil }\end{array}$ & \begin{tabular}{|c|} 
Toca do Garrincho \\
(35610) \\
Brésil
\end{tabular} & & \\
\hline Long. Totale & 346,8 & 350,2 & 408,2 & 395,7 & 465 & 374,8 \\
\hline DAP prox.art. & 87,3 & 86,4 & 85,3 & - & 100 & $84(?)$ \\
\hline DT prox.max. & 171,2 & 170,7 & 210,5 & 208,1 & 233 & 184,7 \\
\hline DAP dist.art. & 103,1 & 104,7 & 110,2 & - & 126 & 94,1 \\
\hline DT dist.art. & 137,8 & 138,4 & 140 & 147,6 & 176 & 134,6 \\
\hline
\end{tabular}

Tab. 5 : Scelidodon - Comparaison des mesures du fémur - dimensions en $\mathbf{m m}$.

Tab. 5: Scelidodon - Comparison of the measurements of femur - dimensions in mm.
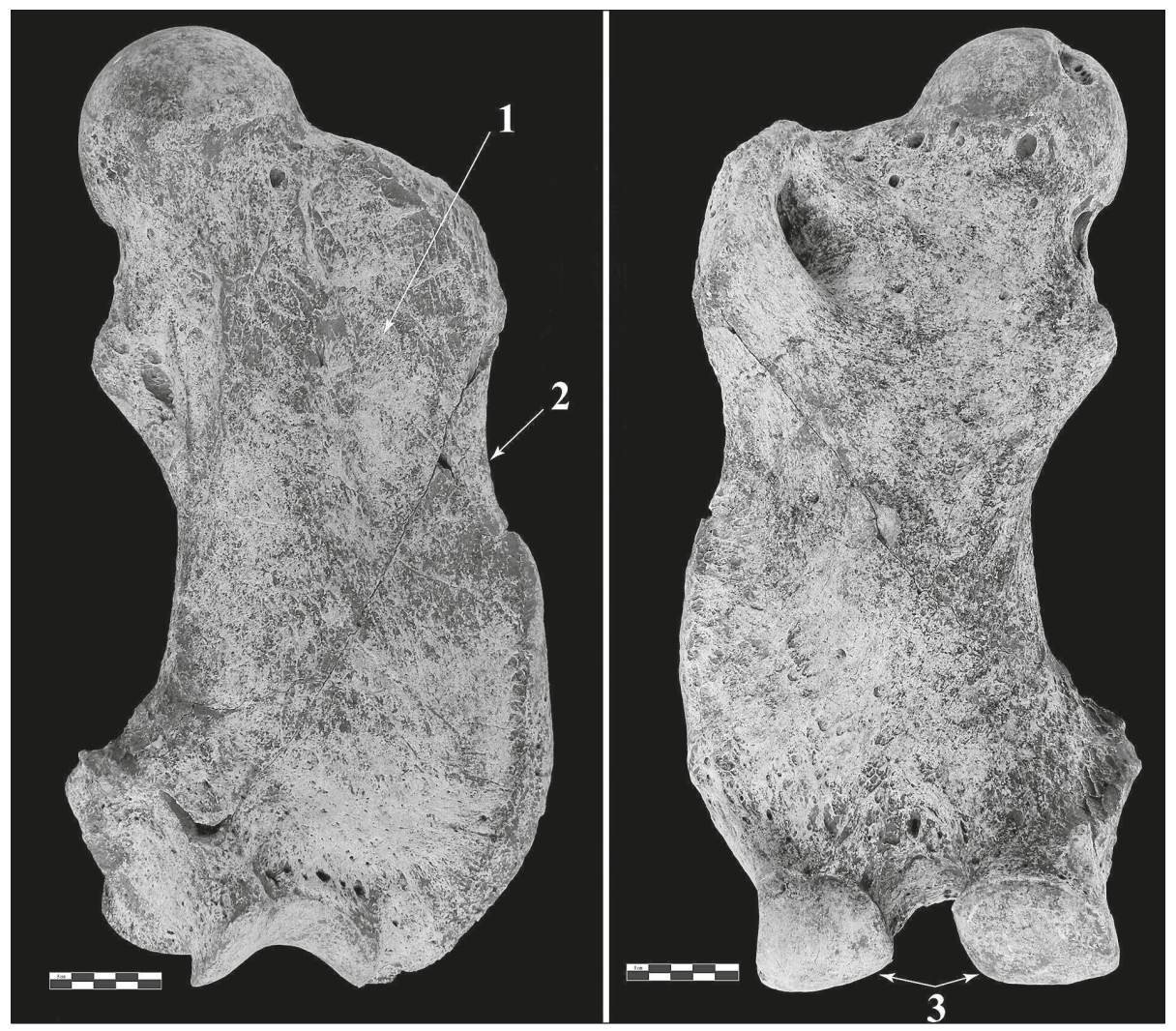

Fig. 7 : Fémur gauche de Scelidodon spécimen $\mathrm{n}^{\circ} 1$ (FUMDHAM-n $\left.{ }^{\circ} 194759\right)$.

À gauche: vue antérieure; à droite : vue postérieure. 1 / surface aplanie ; 2 / ensellure bien marquée ; 3 / condyles distaux sub-égaux.

Fig. 7: Left femur of Scelidodon specimen $n^{\circ} 1$ (FUMDHAM- $\left.n^{\circ} 194759\right)$ Left : anterior view; right : posterior view. 1/ flattened surface; 2/ concavity: 3/ sub-equal distal condyles.

légèrement plus grands que ceux de $S$. chiliensis recueillis à Taramugal - Chili (Guérin \& Faure, 2004 - cf. supra), le fémur de $S$. chiliensis mis au jour à Pampa de los Fósiles se distingue par une longueur et des proportions bien plus importantes que celles des spécimens brésiliens. Pujos écrit d'ailleurs au sujet du Scelidodon de la Pampa des los Fósiles que « cet animal est le plus grand $S$. chiliensis recensé à ce jour » (Pujos, 2000, p. 203). Ceci semblerait attester, comme nous l'avons pressenti lors de l'étude de l'humérus, que les Scelidotheriinae se caractérisent par un dimorphisme sexuel important : les mâles étant bien plus grands que les femelles.

Les fémurs du spécimen $\mathrm{n}^{\circ} 1$ de la Toca da Pena sont, quant à eux, bien plus petits et moins robustes que tous les fémurs de Scelidodon auxquels nous les avons comparés (tab. 5). Cela semblerait confirmer que notre spécimen, par sa petite taille, pourrait être une femelle et que les spécimens provenant de la Toca do Barrigudo et de la Toca do Garrincho seraient des mâles.

D'un point de vue morphologique, les fémurs de la
Toca da Pena sont parfaitement identiques à ceux qui sont attribués au genre Scelidodon et se distinguent très nettement de celui qui se rapporte au genre Catonyx (fig. 7) :

- ils présentent, au niveau de la diaphyse, sur la face antérieure, juste à côté du grand trochanter, une surface relativement plane. Sur le fémur de Catonyx, cette zone présente une forte dépression;

- en vue antérieure, sur le bord latéral, il y a une ensellure bien marquée au milieu de la diaphyse. Chez Catonyx, le bord latéral est plus régulier ;

- les deux condyles distaux sont de taille sensiblement égale, alors que chez Catonyx, le condyle latéral est beaucoup plus petit.

\section{5 - LES AUTRES OS DE SCELIDODON - SPÉCIMEN N 1}

Nous disposons, pour le spécimen $n^{\circ} 1$, de plusieurs autres os complets. D'un point de vue morphologique, ces os sont tout à fait comparables à ceux de Scelidodon et se distinguent très nettement de ceux de Catonyx. Sur 


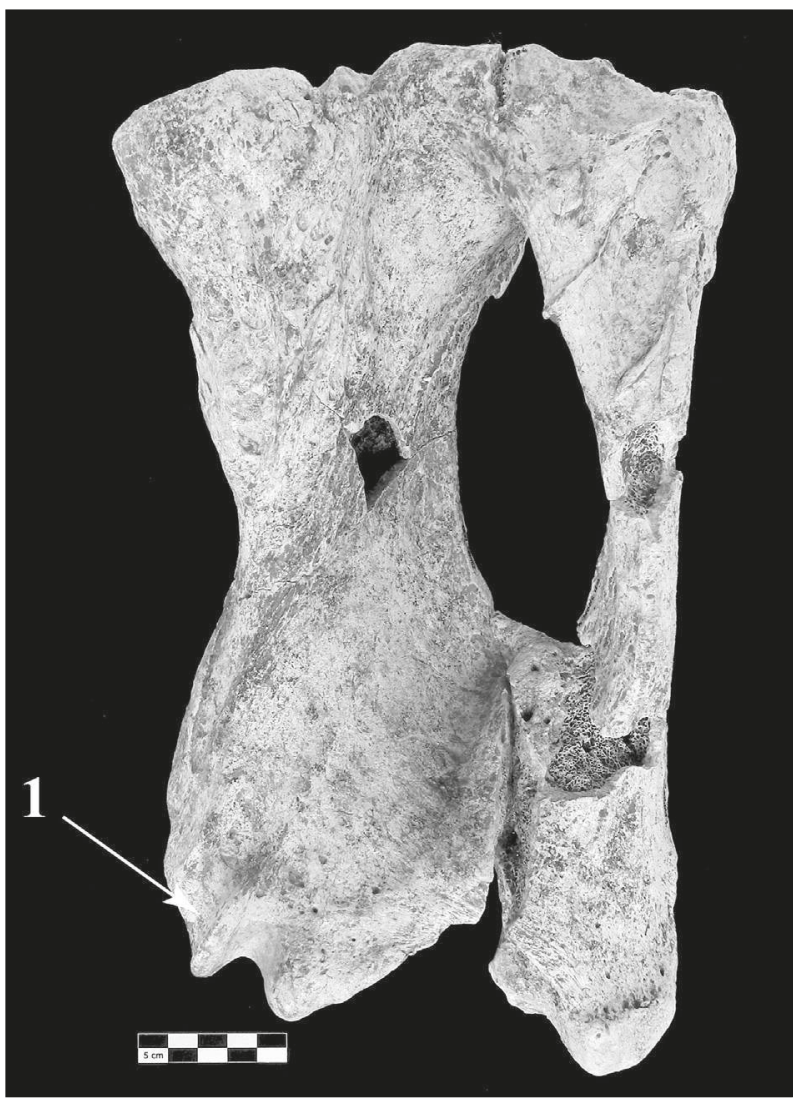

Fig. 8 : Tibia droit de Scelidodon - spécimen n 1 (FUMDHAM-n²02063) vue postérieure. 1 : absence d'un pont osseux.

Fig. 8: Right tibia of Scelidodon - specimen $n^{\circ} 1$ (FUMDHAM- $\left.{ }^{\circ} 202063\right)$ posterior view. 1: absence of a bone bridge.

le tibia, notamment, l'absence d'un pont osseux incomplet au niveau de l'insertion du muscle semimembranosus est caractéristique du genre Scelidodon (Pujos, 2000) (fig. 8).

Les mesures biométriques prises sur la scapula, le radius, l'ulna, le tibia, le talus et la pattela du spécimen $\mathrm{n}^{\circ} 1$ sont reportées dans les tableaux 6 à 11, aux côtés des mesures des os homologues de $S$. piauiense et de S. chiliensis. Il ressort de l'examen de ces tableaux que le spécimen de la Toca da Pena présente des proportions des principaux os du squelette appendiculaire comparables à celle de $S$. piauiense. Il est tout de fois un peu plus petit que les autres fossiles brésiliens de Scelidodon.

\section{6 - SCELIDODON - SPÉCIMEN N²}

Le spécimen $\mathrm{n}^{\circ} 2$ est nettement moins complet que le spécimen $\mathrm{n}^{\circ} 1$ et ses os ont été retrouvés dispersés dans la moitié inférieure de la séquence fouillée. Par ailleurs, la plupart d'entre eux ont été brisés et déformés par le poids des sédiments sus-jacent.

Parmi les éléments dont nous disposons, seuls les tibias permettent d'attribuer avec certitude le spécimen $\mathrm{n}^{\circ} 2$ à Scelidodon. En effet, dans la région postéro-distale de la diaphyse, il n'y a pas d'excroissance osseuse permettant l'insertion du muscle semimembranosus, contrairement à ce que l'on peut observer chez Catonyx (Pujos, 2000).

Les os longs du spécimen $n^{\circ} 2$ ne sont pas épiphysés. Il s'agit donc d'un animal juvénile. L'état de conserva- tion des os de cet animal ne permet pas de réaliser une étude biométrique. Cependant lorsque l'on compare, en les mettant côte à côte, les différents éléments osseux du spécimen $n^{\circ} 1$ avec ceux du spécimen $n^{\circ} 2$, ils paraissent plus grands et plus robustes chez ce dernier, alors qu'il n'avait pas fini sa croissance. Cela traduit donc des différences de taille importantes au sein de l'espèce Scelidodon piauiense, pouvant être le reflet d'un dimorphisme sexuel.

L'ensemble des caractères morphologiques observés sur les os du Scelidotheriinae de la Toca da Pena, se distinguent très nettement de ceux se rapportant au genre Catonyx et sont, au contraire, comparables à ceux observés chez Scelidodon. Le crâne qui présente une région occipitale plus basse que chez $S$. chiliensis, est comparable à celui de $S$. piauiense, espèce à laquelle nous le repportons. Cependant, le spécimen $\mathrm{n}^{\circ} 1$ de la Toca da Pena est plus petit que les autres représentants brésiliens de $S$. piauiense.

L'étude biométrique a montré qu'il existait, aussi bien chez $S$. piauiense que chez $S$. chiliensis, des différences de taille, parfois importantes, entre les différents spécimens recencés. Ces différences de taille ne peuvent correspondre qu'à un fort dimorphisme sexuel au sein de ces deux espèces de Scelidodon : les mâles devant être environ $15 \%$ plus grands que les femelles.

La différence de taille observée entre les deux spécimens mis au jour dans la Toca da Pena confirme cette hypothèse. En effet, les ossements rapportés au Scelidodon $\mathrm{n}^{\circ} 2$ présentent des dimensions plus grandes que ceux du spécimen $n^{\circ} 1$. Il s'agit pourtant d'un individu juvénile qui n'avait donc pas fini sa croissance au moment de sa mort. Nous en faisons, par conséquent, un jeune mâle et le spécimen $n^{\circ} 1$ serait une femelle adulte. De ce fait, nous rajoutons deux individus supplémentaires à la liste se rapportant à l'espèce Scelidodon piauiense. Espèce qui, à ce jour, n'a été identifiée que dans le sud-est du Piauí.

\section{3 - LES AUTRES TAXONS IDENTIFIÉS À LA TOCA DA PENA}

\section{1 - LES DASYPODIDÉS - DASYPUSNOVEMCINC- TUS, TOLYPEUTES TRICINCTUS ET EUPHRACTUS SEXCINCTUS}

À la Toca da Pena, seuls des tatous de la famille des Dasypodidés ont été identifiés. Ils sont représentés principalement par des ostéodermes. Leur morphologie et leur ornementation permettent de reconnaître trois espèces : Dasypus novemcinctus, Tolypeutes tricinctus et Euphractus sexcinctus ; ce dernier ayant livré le plus grand nombre de restes. Outre les ostéodermes, nous avons également rapporté à cette espèce : un fémur, un fragment proximal de tibia, un pelvis et un calcanéum.

Ces trois espèces font partie de la faune actuelle du Brésil (dos Reis et al., 2011). Les enregistrements fossiles indiquent que Dasypus novemcinctus et Tolypeutes tricinctus sont présents dans ce pays dès le début du 


\begin{tabular}{|l|c|c|c|}
\cline { 2 - 4 } \multicolumn{1}{c|}{} & \multicolumn{2}{c|}{ Scelidodon piauiense } & $\begin{array}{c}\text { Scelidodon chiliensis } \\
\text { (Pujos, 2000) }\end{array}$ \\
\hline Scapula & $\begin{array}{c}\text { Toca da Pena } \\
(194743) \\
\text { Brésil }\end{array}$ & $\begin{array}{c}\text { Toca do Barrigudo } \\
(79099)\end{array}$ & Pampa de los Fosiles \\
Brésil & Pérou \\
\hline DAP zone art. & 70,4 & 79,4 & 105 \\
DT zone art. & - & - & 60 \\
DT palette max. & 353,2 & 302,6 & - \\
HT le long de la crête scapulaire & 350,4 & $369(?)$ & - \\
\hline
\end{tabular}

Tab. 6 : Scelidodon - Comparaison des mesures de la scapula - dimensions en mm.

Tab. 6: Scelidodon-Comparison of the measurements of scapula-dimensions in mm.

\begin{tabular}{|l|c|c|c|c|}
\cline { 2 - 4 } \multicolumn{1}{c|}{} & \multicolumn{3}{c|}{ Scelidodon piauiense } \\
\hline Radius & $\begin{array}{c}\text { Toca da Pena } \\
(179445) \\
\text { Brésil }\end{array}$ & $\begin{array}{c}\text { Toca da Pena } \\
(179445) \\
\text { Brésil }\end{array}$ & $\begin{array}{c}\text { Toca da Pena } \\
(167890) \\
\text { Brésil }\end{array}$ & $\begin{array}{c}\text { Toca do Barrigudo } \\
(79159) \\
\text { Brésil }\end{array}$ \\
\hline Long. Totale & - & - & - & - \\
HT art.prox. & 44,1 & 43,6 & - & - \\
DT prox.art. & 51 & 51,8 & 61,3 \\
DAP dist.max. & - & - & 84,5 \\
DT dist.max. & - & - & 63,2 \\
\hline
\end{tabular}

Tab. 7 : Scelidodon - Comparaison des mesures du radius - dimensions en mm.

Tab. 7: Scelidodon-Comparison of the measurements of radius - dimensions in mm.

\begin{tabular}{|c|c|c|c|}
\hline \multirow[b]{2}{*}{ Ulna } & \multicolumn{3}{|c|}{ Scelidodon piauiense } \\
\hline & $\begin{array}{c}\text { Toca da Pena } \\
(180500) \\
\text { Brésil }\end{array}$ & $\begin{array}{c}\text { Toca da Pena } \\
(180435) \\
\text { Brésil }\end{array}$ & $\begin{array}{c}\text { Toca do Barrigudo } \\
\text { (79244) } \\
\text { Brésil }\end{array}$ \\
\hline Long. Totale & 346 & - & 408 \\
\hline HT art.prox. & 61,6 & - & 60,6 \\
\hline DT prox.art. & 82,6 & - & 89,7 \\
\hline DAP dist.art. & 28,1 & 27,6 & 33,7 \\
\hline DT dist.art. & 42,4 & 41,6 & 46,6 \\
\hline DT dist.max. & 45,6 & 46,2 & 48,9 \\
\hline
\end{tabular}

Tab. 8 : Scelidodon - Comparaison des mesures de l'ulna - dimensions en mm.

Tab. 8: Scelidodon - Comparison of the measurements of ulna-dimensions in mm.

\begin{tabular}{|l|c|c|c|c|}
\cline { 2 - 5 } \multicolumn{1}{c|}{} & \multicolumn{3}{c|}{ Scelidodon piauiense } & $\begin{array}{c}\text { Scelidodon chiliensis } \\
\text { (Pujos, 2000) }\end{array}$ \\
\hline Tibia & $\begin{array}{c}\text { Toca da Pena } \\
\text { (201462) } \\
\text { Brésil }\end{array}$ & $\begin{array}{c}\text { Toca do Barrigudo } \\
\text { (79373) }\end{array}$ & $\begin{array}{c}\text { Toca do Garrincho } \\
\text { (37532) } \\
\text { Brésil }\end{array}$ & Pampa de los Fosiles \\
\hline Long. Totale & 279,3 & 341,2 & 308,3 & 290 \\
DAP prox.max. & 103,8 & 120,8 & 110,6 & 137 \\
DT prox.max. & 162,1 & 183,6 & 168,3 & 173 \\
DT dist.max. & 150,3 & 120,8 & 162,4 & 154 \\
\hline
\end{tabular}

Tab. 9 : Scelidodon - Comparaison des mesures du tibia - dimensions en mm.

Tab. 9: Scelidodon - Comparison of the measurements of tibia-dimensions in mm.

\begin{tabular}{|l|c|c|c|c|}
\cline { 2 - 5 } \multicolumn{1}{c|}{} & \multicolumn{3}{c|}{$\begin{array}{c}\text { Scelidodon chiliensis } \\
\text { (Pujos, 2000) }\end{array}$} & Pcelidodon piauiense \\
\hline \multirow{2}{*}{ Talus } & $\begin{array}{c}\text { Toca da Pena } \\
(234012) \\
\text { Brésil }\end{array}$ & $\begin{array}{c}\text { Toca da Pena } \\
(20146)\end{array}$ & $\begin{array}{c}\text { Toca do Barrigudo } \\
(79281) \\
\text { Brésil }\end{array}$ & Pérou \\
\hline DAP & 93,7 & Brésil & 100,5 & 103 \\
DT & 89,8 & 92,5 & 93,8 & 100 \\
HT & 57,4 & 88,9 & 66,2 & - \\
\hline
\end{tabular}

Tab. 10 : Scelidodon - Comparaison des mesures du talus - dimensions en mm.

Tab. 10: Scelidodon - Comparison of the measurements of talus - dimensions in mm.

\begin{tabular}{|l|c|c|}
\cline { 2 - 3 } \multicolumn{1}{c|}{} & Scelidodon piauiense & $\begin{array}{c}\text { Scelidodon chiliensis } \\
\text { (Pujos, 2000) }\end{array}$ \\
\hline Patella & $\begin{array}{c}\text { Toca da Pena } \\
(167858)\end{array}$ & Pampa de los Fosiles \\
& Brésil & Pérou \\
\hline DAP & 49,3 & - \\
DT & 76 & 86 \\
HT & 113 & - \\
\hline
\end{tabular}

Tab. 11 : Scelidodon - Comparaison des mesures de la patella - dimensions en mm.

Tab. 11: Scelidodon-Comparison of the measurements of patella-dimensions in mm. 
Pléistocène, alors que Euphractus sexcinctus n'apparait qu'à partir du Pléistocène récent (Soibelzon et al., 2015).

\section{2 - LES FÉLIDÉS - LEOPARDUS PARDALIS}

Les Félidés sont représentés à la Toca da Pena par un total de 88 restes. La majorité d'entre eux $(\mathrm{NR}=72)$ sont rapportés à l'ocelot, Leopardus pardalis. Ils correspondent à quelques vertèbres, des fragments diaphysaires d'os longs et surtout aux petits os de l'autopode. La pièce la plus remarquable est un fragment de la branche horizontale d'une mandibule gauche où sont encore présentes la $\mathrm{P}_{3}$, la $\mathrm{P}_{4}$ et la $\mathrm{M}_{1}$. Les dimensions de ces dents sont reportées dans le tableau 12 .

\begin{tabular}{|l|c|}
\cline { 2 - 2 } \multicolumn{1}{c|}{} & Leopardus pardalis \\
\hline Mandibule & $\begin{array}{c}\text { Toca da Pena } \\
(211265) \\
\text { Brésil }\end{array}$ \\
\hline DMD P3 & 8,5 \\
DMD P4 & 11,4 \\
DMD M1 & 12,7 \\
\hline
\end{tabular}

Tab. 12 : Leopardus pardalis - Comparaison des mesures diamètre mésio-distal (DMD) des dents jugales inférieures - dimensions en mm. Tab. 12: Leopardus pardalis - Comparison of meso-distal diameter (DMD) of lower jugular tooth - dimensions in $\mathrm{mm}$.

Un second Félidé, plus petit que l'ocelot, est également présent dans l'assemblage faunique de la Toca da Pena $(\mathrm{NR}=16)$. Il n'est représenté que par quelques restes et leur état de conservation ne nous a pas permis une attribution taxonomique.

\section{3 - LES ÉQUIDÉS - HIPPIDION SP.}

Dans les sites pléistocènes et holocènes anciens du Nordeste du Brésil, les Équidés sont representés par Equus et Hippidion, ce dernier étant souvent le plus abondant (Guérin \& Faure, 2008).

Dans l'assemblage faunique de la Toca da Pena, les Équidés ne sont représentés que par trois tarsiens fragmentés et une deuxième prémolaire supérieure. Les tarsiens, en raison de leur état de conservation, ne permettent pas une détermination au niveau du genre. En revanche, la prémolaire, qui présente une couronne relativement basse (Alberdi \& Prado, 1992 ; Prado \& Alberdi, 1996) est rapportée à Hippidion.

\section{4 - LES TAYASSUIDÉS - TAYASSU PECARI}

Dans les sites paléontologiques du Brésil, trois espèces de Tayassuidés ont été identifiées : Tayassu tajacu, Tayassu pecari et Catagonus stenocephalus (Parisi Dutra et al., 2016), mais seules les deux premières ont été identifiées dans l'état du Piauí (Guérin et al., 1993a,b ; Faure et al., 1999 ; Guérin \& Faure, 2008)

Tous les restes de Tayassuidés recueillis à la Toca da Pena correspondent à des fragments de maxillaires ou de mandibules et à des dents isolées. Aucun élément du squelette post-crânien n'a été identifié. La plupart des dents sont endommagées ou se rapportent à des individus juvéniles, ce qui n'a pas permis de réaliser une étude biométrique. Cependant, tous ces restes présentent de fortes similitudes avec les spécimens de Tayassu pecari de la collection ostéologique de la FUMDHAM auquel nous les avons comparés. Nous rapportons donc tous les restes de Tayassuidés de la Toca da Pena à l'espèce Tayassu pecari.

\section{5 - LE CAMÉLIDÉ - PALAEOLAMA NIEDAE}

Dans les sites pléistocènes du Nordeste du Brésil, les restes de Camélidés correspondent principalement au genre Palaeolama, représenté par deux espèces :

- Palaeolama major (Liais, 1872) : Il est présent au Brésil dans des sites du Pléistocène supérieur et de l'Holocène ancien. Il se caractérise par des membres longs et graciles.

- Palaeolama niedae Guérin \& Faure, 1999 : Il est plus abondant que le précédent dans les sites du Nordeste brésilien datant de la fin du Pléistocène et perdure jusqu'à l'Holocène ancien. C'est une forme de très grande taille, moins gracile que $P$. major et qui présente des dents jugales plus courtes que $P$. major.

À la Toca da Pena, la présence du genre Palaeolama est attestée par 25 restes osseux, dont une grande partie a été recueillie dans le secteur de la grotte. Beaucoup correspondent à des fragments diaphysaires. Seuls une $\mathrm{P} 4$ supérieure, un fragment de mandibule avec $\mathrm{M}_{1}$ et $\mathrm{M}_{2}$ et un métatarsien complet permettent une détermination au rang de l'espèce. Les dents jugales sont relativement courtes et le métatarsien est très grand (tab. 13). Ces différents éléments squelettiques se rapprochent donc, par leurs dimensions, de ceux de Palaeolama niedae.

Par conséquent, comme dans les autres sites du Sud-Est du Piauí datant du Pléistocène supérieur : Toca do Garrincho et Toca da Janela da Barra do Antonião (Guérin \& Faure, 1999), c'est l'espèce de très grande taille, Palaeolama niedae qui est présente à la Toca da Pena.

\section{6 - LES CERVIDÉS - MAZAMA AMERICANA ET} MAZAMA GOUAZOUBIRA

Trois espèces de Cervidés sont attestées dans le Pléistocène supérieur et l'Holocène ancien du sud-est du Piauí : le Daguet gris, Mazama gouazoubira et le grand Daguet, Mazama americana, qui font toujours partie de la faune de la région, et le Cerf des marais, Blastocerus dichotomus, qui en a actuellement disparu (Guérin \& Faure, 2009 ; dos Reis et al., 2011).

À la Toca da Pena, 30 restes osseux ont été rapportés à la famille des Cervidés. Deux fragments de mandibule, une première molaire supérieure et une épiphyse proximale de métacarpien ont été comparés, d'un point de vue biométrique, avec les trois espèces de Cervidés possibles (tab. 14). Un fragment de mandibule avec encore $\mathrm{P}_{4}$ et $\mathrm{M}_{1}$ a été attribué à $M$. americana. Les autres restes mesurés se rapportent, quant à eux, à M. gouazoubira. 


\begin{tabular}{|c|c|c|c|c|c|c|c|c|c|}
\hline & Toca da Pena & \multicolumn{4}{|c|}{$\begin{array}{c}\text { Palaeolama niedae } \\
\text { (Guérin \& Faure 1999) }\end{array}$} & & \multicolumn{3}{|c|}{$\begin{array}{c}\text { Palaeolama major } \\
\text { (Guérin \& Faure 1999) }\end{array}$} \\
\hline \multicolumn{10}{|c|}{ Fragment mandibulaire avec M1 et M2 inférieure (180515) } \\
\hline \multicolumn{2}{|l|}{ M1 } & $\mathrm{n}$ & $\mathrm{x}$ & $\min$ & $\max$ & $\mathrm{n}$ & $\mathrm{x}$ & $\min$ & $\max$ \\
\hline DMD & 18 & 4 & 18,75 & 17,5 & 20 & 4 & 21 & 18 & 23,5 \\
\hline DVL & 14,7 & 4 & 12,88 & 12,5 & 13,5 & 4 & 13,63 & 13 & 15 \\
\hline \multicolumn{2}{|l|}{ M2 } & $\mathrm{n}$ & $\mathrm{x}$ & $\min$ & $\max$ & $\mathrm{n}$ & $\mathrm{x}$ & $\min$ & $\max$ \\
\hline DMD & 19,6 & 13 & 21,35 & 18 & 29,5 & 3 & 23,83 & 21 & 28 \\
\hline DVL & 15,6 & 12 & 13,75 & 12 & 15,5 & 3 & 14,17 & 13,5 & 14,5 \\
\hline \multicolumn{10}{|c|}{ P3 supérieure (169023) } \\
\hline \multirow{3}{*}{$\begin{array}{l}\text { DMD } \\
\text { DVL }\end{array}$} & & $\mathrm{n}$ & $\mathrm{x}$ & $\min$ & $\max$ & $\mathrm{n}$ & $\mathrm{x}$ & $\min$ & $\max$ \\
\hline & 15,1 & 8 & 16,94 & 15 & 20 & 1 & 17,5 & - & - \\
\hline & 16,2 & 8 & 14,63 & 13 & 15,5 & 1 & 18 & - & - \\
\hline \multicolumn{10}{|c|}{ Métatarsien (234244) } \\
\hline \multirow[b]{2}{*}{ Long. totale } & & $\mathrm{n}$ & $\mathrm{x}$ & $\min$ & $\max$ & $\mathrm{n}$ & $\mathrm{x}$ & $\min$ & $\max$ \\
\hline & 338,2 & 7 & 310,14 & 300 & 336 & 5 & 288,4 & 281 & 301 \\
\hline
\end{tabular}

Tab. 13 : Palaeolama - Comparaison des mesures des dents et du métatarsien - dimensions en mm.

Tab. 13: Palaeolama - Comparison of measurements of teeth and metatarsal -dimensions in mm.

\begin{tabular}{|c|c|c|c|c|c|c|c|c|c|c|c|c|c|}
\hline \multicolumn{2}{|c|}{ Toca da Pena } & \multicolumn{4}{|c|}{$\begin{array}{l}\text { Mazama gouazoubira } \\
\text { Actuel } \\
\text { (Guérin \& Faure, 2009) }\end{array}$} & \multicolumn{4}{|c|}{$\begin{array}{l}\text { Mazama americana } \\
\text { Actuel } \\
\text { (Guérin \& Faure, 2009) }\end{array}$} & \multicolumn{4}{|c|}{$\begin{array}{c}\text { Blastocerus dichotomus } \\
\text { Actuel } \\
\text { (Guérin \& Faure, 2009) }\end{array}$} \\
\hline \multicolumn{2}{|c|}{$\begin{array}{l}\text { Mandibule - fragment avec D4-M1 } \\
(167854)\end{array}$} & \multirow[b]{2}{*}{$\mathrm{n}$} & \multirow[b]{2}{*}{$\mathrm{x}$} & \multirow[b]{2}{*}{$\min$} & \multirow[b]{2}{*}{$\max$} & \multirow[b]{2}{*}{$\mathrm{n}$} & \multirow[b]{2}{*}{$\mathrm{x}$} & \multirow[b]{2}{*}{$\min$} & \multirow[b]{2}{*}{$\max$} & \multirow[b]{2}{*}{$\mathrm{n}$} & \multirow[b]{2}{*}{$\mathrm{x}$} & \multirow[b]{2}{*}{$\min$} & \multirow{2}{*}{$\min$} \\
\hline Mazama gou & & & & & & & & & & & & & \\
\hline Haut. mand. D4-M1 & 12,4 & 32 & 14,7 & 12,0 & 18,5 & 28 & 17,6 & 14,5 & 22,5 & 4 & 24,4 & 20,5 & 26,0 \\
\hline DT mand. P4-M1 & 7,6 & 32 & 7,6 & 6,0 & 8,5 & 28 & 9,8 & 7,5 & 11,5 & 4 & 13,7 & 12,0 & 17,0 \\
\hline \multicolumn{2}{|c|}{$\begin{array}{l}\text { Mandibule - fragment avec } \\
\text { M2-M3 (234693) }\end{array}$} & \multirow[b]{2}{*}{$\mathrm{n}$} & \multirow[b]{2}{*}{$\mathrm{x}$} & \multirow[b]{2}{*}{$\min$} & \multirow[b]{2}{*}{$\max$} & \multirow[b]{2}{*}{$\mathrm{n}$} & \multirow[b]{2}{*}{$\mathrm{x}$} & \multirow[b]{2}{*}{$\min$} & \multirow[b]{2}{*}{$\max$} & \multirow[b]{2}{*}{$\mathrm{n}$} & \multirow[b]{2}{*}{$\mathrm{x}$} & \multirow[b]{2}{*}{$\min$} & \multirow[b]{2}{*}{$\max$} \\
\hline Mazama ame & & & & & & & & & & & & & \\
\hline Haut. mand. M2-M3 & 20,8 & 32 & 16,5 & 14,5 & 20,4 & 28 & 21,0 & 17,0 & 24,5 & 4 & 28,0 & 24,0 & 30,0 \\
\hline DT mand. M2-M3 & 14,3 & 32 & 8,8 & 7,5 & 11,0 & 28 & 11,1 & 8,0 & 13,0 & 4 & 14,9 & 13,0 & 18,0 \\
\hline \multicolumn{2}{|c|}{ M1 supérieure (235053) } & \multirow[b]{2}{*}{$\mathrm{n}$} & \multirow[b]{2}{*}{$\mathrm{x}$} & \multirow[b]{2}{*}{$\min$} & \multirow[b]{2}{*}{$\max$} & \multirow[b]{2}{*}{$\mathrm{n}$} & & & & & & & \\
\hline Mazama gou & & & & & & & $\mathrm{x}$ & $\min$ & $\max$ & $\mathrm{n}$ & $\mathrm{x}$ & $\min$ & $\max$ \\
\hline DMD max & 8,9 & 41 & 10,0 & 8,5 & 12,0 & 40 & 11,8 & 10,0 & 14,0 & 5 & 16,3 & 14,5 & 17,0 \\
\hline
\end{tabular}

Tab. 14 : Cervidae - Comparaison des mesures des dents et du métacarpien - dimensions en $\mathbf{m m}$.

Tab. 14: Cervidae - Comparison of measurements of teeth and metacarpal - dimensions in mm.

\section{4 - DISCUSSION - CONCLUSIONS}

Le site de la Toca da Pena constitue un site majeur pour le Pléistocène supérieur de la région Sud-Est du Piauí. Il est situé dans un massif calcaire, ce qui a permis la conservation d'une faune variée, correspondant à 19 taxons différents : un amphibien, trois reptiles et 15 mammifères. Ce site a également livré une industrie lithique constituée de quelques galets de quartzite taillés.

La faune de la Toca da Pena est dominée par Scelidodon piauiense. Cette espèce, identifié à ce jour seulement dans le Nordeste du Brésil, dans l'état du Piauí, reste rare dans les sites paléontologiques et nous connaissons peu de choses sur ces animaux.

\section{1 - DIMORPHISME SEXUEL CHEZ SCELIDODON}

À la Toca da Pena, nous disposons des ossements de deux individus : un adulte quasiment complet et un jeune adulte. En effet, ce dernier n'avait pas fini sa croissance : ses os longs n'étant pas épiphysés. Malgré cela, ils sont plus grands et plus robustes que ceux du spécimen adulte. Cette différence de taille montre que l'espèce Scelidodon piauiense présentait un dimorphisme sexuel relativement prononcé : les mâles étant $15 \%$ plus grands que les femelles. Ce dimorphisme sexuel peut être étendu à l'ensemble des espèces se rapportant au genre Scelidodon. Cela permettrait d'expliquer les différences de taille importantes que nous avons constatées entre les différents spécimens de Scelidodon chiliensis, lors de l'étude biométrique réalisée dans le cadre de cet article.

\section{2 - POSITION CHRONOLOGIQUE DE LA FAUNE DE LA TOCA DA PENA}

Scelidodon piauiense est une espèce du Pléistocène supérieur et de l'Holocène ancien. Elle est associée à d'autres espèces : Palaeolama niedae, Hippidion sp., 


\begin{tabular}{|c|c|c|c|c|c|c|}
\hline Site & Secteur & Niveau & Méthode & Code labo. & $\begin{array}{c}\text { Age brut } \\
\text { (non calibré) BP }\end{array}$ & $\begin{array}{c}\text { Age calibré } \\
\text { (années cal. BP) }\end{array}$ \\
\hline La Pena & Secteur nord & $\begin{array}{c}\text { carré H19 } \\
\mathrm{z}=3,65 \mathrm{~m}\end{array}$ & ${ }^{14} \mathrm{C}$ & $\begin{array}{c}\text { SacA } 17458 \\
\text { Gif-12438 }\end{array}$ & $10020 \pm 50$ & {$[11305-11752](1.00)$} \\
\hline La Pena & Secteur sud & $\begin{array}{c}\text { locus sud } \\
\mathrm{z}=-1,95 \mathrm{~m}\end{array}$ & ${ }^{14} \mathrm{C}$ & $\begin{array}{c}\text { SacA } 17459 \\
\text { Gif-12439 }\end{array}$ & $10210 \pm 60$ & $\begin{array}{l}{[11639-11672](0.02)} \\
{[11698-12142](0.98)}\end{array}$ \\
\hline La Pena & Grotte & $\begin{array}{c}\mathrm{E} 12 / \mathrm{sc} 1 \\
\mathrm{z}=1,88 \mathrm{~m}\end{array}$ & ${ }^{14} \mathrm{C}$ & $\begin{array}{c}\text { Gif } 13105 \\
\text { SacA37778 }\end{array}$ & $9480 \pm 20$ & $\begin{array}{l}{[10582-10832](0.75)} \\
{[10840-10868](0.03)} \\
{[10951-11070](0.22)}\end{array}$ \\
\hline
\end{tabular}

Tab. 15 : Résultats des datations ${ }^{14} \mathrm{C}$ réalisées sur charbons.

Tab. 15: Results of ${ }^{14} \mathrm{C}$ datings performed on charcoals.

toutes deux présentes pendant le Pléistocène supérieur et qui disparaissent au cours de l'Holocène ancien.

Par ailleurs, nous disposons de trois datations ${ }^{14} \mathrm{C}$ effectuées sur charbons : deux ont été prélevés dans l'abri sous roche qui prolonge la grotte et le troisième dans la grotte (tab. 15). Les résultats de ces datations ${ }^{14} \mathrm{C}$ permettent d'affiner la datation relative obtenue à partir des données paléontologiques : le site de la Toca da Pena est donc contemporain de la transition Pléistocène supérieur - Holocène.

\section{3 - INTERPRÉTATIONS PALÉOENVIRONNE- MENTALES}

À la Toca da Pena, au côté des espèces éteintes : Scelidodon piauiense, Palaeolama niedae et Hippidion sp., ont été identifiées des espèces toujours présentes dans la faune actuelle : Leopardus pardalis, Tayassu pecari, Mazama americana et Mazama gouazoubira.

Cette association faunique témoigne d'une formation végétale correspondant à une forêt sèche, constituée d'une strate arborescente plus au moins continue et d'un tapis graminéen couvrant partiellement le sol (selon la définition de Bellefontaine et al., 1997). Cette végétation se rapproche davantage du cerrado que de la caatinga actuelle. Cela implique, à la fin du Pléistocène dans l'État du Piauí, des conditions climatiques moins sèches qu'aujourd'hui (Guérin \& Faure, 2008, 2014).

\section{REMERCIEMENTS}

Nous remercions le Ministère français des Affaires étrangères pour le financement annuel de la Mission franco-brésilienne au Piauí. Nous remercions également la FUMDHAM qui nous a accueilli dans ses locaux, lors de notre séjour à São Raimundo Nonato, pour nous permettre de consulter les collections ostéologiques et paléontologiques.

Nous tenons tout particulièrement à remercier Claude Guérin et Martine Faure pour les discussions et leurs conseils. Nous avons appris beaucoup auprès d'eux, mais cela représente bien peu à côté de l'immense connaissance qu'ils ont des faunes fossiles d'Amérique du Sud. Nous les remercions également pour le temps qu'ils ont consacré à nous faire visiter les principaux sites paléontologiques de la région de São Raimundo Nonato.
La disparition de Claude Guérin constitue une grande perte pour le vaste monde de la paléontologie et de l'archéologie, qui ne se limitait pas à l'Amérique du Sud.

\section{RÉFÉRENCES BIBLIOGRAPHIQUES}

ALBERDI M.T. \& PRADO J.L. 1992 - El registro de Hippidion Owen, 1869 y Equus (Amerhippus) Hoffstetter, 1950 (Mammalia, Perissodactyla) en América del Sur. Ameghiniana, 29, 265-284.

BELLEFONTAINE R., GASTON A. \& PETRUCCI Y., 1997 - Aménagement des forêts naturelles des zones tropicales sèches. Cahier FAO Conservation, 32. Cirad, Montpellier \& FOA, Rome, $315 \mathrm{p}$.

DOS REIS N.R., PERACCHI A.L, PEDRO W.A. \& LIMA I.P

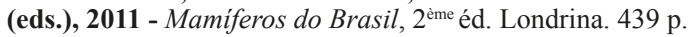

FAURE M., GUÉRIN C. \& PARENTI F., 1999 - Découverte d'une mégafaune holocène à la Toca do Serrote do Artur (aire archéologique de São Raimundo Nonato, Piauí, Brésil). Comptes Rendus de l'Académie des Sciences. Série 2, Sciences de la Terre et des Planètes, 329, 443-448.

GUÉRIN C. \& FAURE M., 1999. Palaeolama (Hemiauchenia) niedae nov. sp., nouveau Camelidae du Nordeste brésilien et sa place parmi les Lamini d'Amérique du Sud. Geobios, 32 (4), 629-659.

GUÉRIN C. \& FAURE M., 2004 - Scelidodon piauiense nov. sp., nouveau Mylodontidae Scelidotheriinae (Mammalia, Xenarthra) du Quaternaire de la région du parc national Serra da Capivara (Piauí, Brésil). Comptes Rendus Palevol, 3 (1), 35-42.

GUÉRIN C. \& FAURE M., 2008 - La biodiversité mammalienne au Pléistocène supérieur - Holocène ancien dans la Région du Parc National Serra da Capivara (SE du Piaú, Brésil). FUMDHAMentos - Revista da Fundação Museu do Homem Americano, 7, 80-93.

GUÉRIN C. \& FAURE M., 2009 - Les Cervidae (Mammalia, Artiodactyla) du Pléistocène supérieur-Holocène ancien de la région du Parc National Serra da Capivara (Piauí, Brésil). Geobios, 42, 169-195.

GUÉRIN C. \& FAURE M., 2014. Paleontologia da região do parque nacional Serra da Capivara. In A.-M. Pessis, G. Martin \& N. Guidon (eds.). Os Biomas e as sociedades humanas na pré-história da região do Parque Nacional Serra da Capivara, Brasil, vol. II-A. A\&A Comunicacão, São Paulo, 142-182.

GUÉRIN C., CURVELLO M.A., FAURE M., HUGUENEY M. \& MOURER-CHAUVIRÉ C., 1993a - La faune pléistocène du Piauí (Nordeste du Brésil) : Implications paléoécologiques et biochronologiques. Quaternaria Nova, 3, 303-341.

GUÉRIN C., HUGUENEY M., MOURER-CHAUVIRÉ C. \& FAURE M., 1993b - Paléoenvironnement pléistocène dans l'aire archéologique de São Raimundo Nonato (Piauí, Brésil) : Apport des Mammifères et des Oiseaux. Documents des Laboratoires de Géologie de Lyon, 125, 187-202.

PARISI DUTRA R., VELLOSO MISSAGIAR., ARAUJO PERINI F., COZZUOL M.A., GASPARINI G.M., GONÇALVES GUEDES P. \& DE OLIVEIRA SALLES L., 2016 - Fossil peccaries of Late Pleistocene/Holocene (Cetartiodactyla, Tayassuidae) from underwater caves of Serra da Bodoquena (Mato Grosso do Sul State, Brazil). Historical Biology, 29 (1), 85-92.

PRADO J.L. \& ALBERDI M.T., 1996 - A cladistic analysis of the Horses of the tribe Equini. Palaeontology, 39, 663-680.

PUJOS F., 2000 - Scelidodon chiliensis (Xenarthra, Mammalia) du Pléistocène terminal de " Pampa de los Fósiles » (Nord-Pérou). Quaternaire, 11 (3-4) 197-206. 
RASSE M., BOËDA E., BINANT P., LOURDEAU A., TEIXEIRA MENDES D. LAHAYE C., GUERIN C. \& FONTUGNE M. 2009 - Mission franco-brésilienne du Piauí. Recherches sur le peuplement pléistocène et holocène du Nordeste (Brésil). Rapport du Ministère des Affaires Étrangères, Paris, 48 p.
SOIBELZON E., AVILLA L.S. \& CASTRO M., 2015 - The cingulates (Mammalia: Xenarthra) from the late Quaternary of northern Brazil: Fossil records, paleoclimates and displacements in America. Quaternary International, 377, 118-125. 